
múltiplas colheitas. Horticultura Brasileira 26: 335-341.

\title{
Variância e média da massa de frutos de abobrinha-italiana em múlti- plas colheitas
}

\author{
Alessandro Dal'Col Lúcio; Ricardo H Carpes; Lindolfo Storck; Sidinei José Lopes; Leandro H Lorentz; \\ André Luiz Paludo \\ UFSM-Depto. Fitotecnia, Campus Universitário, Camobi, 97105-900 Santa Maria-RS; adlucio@smail.ufsm.br, rh.carpes@bol.com.br, \\ lindolfo@smail.ufsm.br, sjlopes@smail.ufsm.br, llorentz@bol.com.br, agropaludo@gmail.com
}

\begin{abstract}
RESUMO
A variância associada à produção em plantas com colheitas múltiplas nem sempre é homogênea, o que compromete a precisão experimental. Com o objetivo de identificar o comportamento da média e da variância da massa dos frutos de abobrinha-italiana (Curcubita pepo) com o passar das colheitas, entre as linhas de cultivo dentro de cada colheita e entre colheitas, e verificar a interferência de diferentes manejos nesse comportamento, conduziu-se um trabalho nas estações sazonais inverno-primavera (I/P) e verão-outono (V/O) 2004/2005. O experimento foi conduzido em dois túneis (T1 e T2), com três linhas de 25 plantas por túnel, com a cultivar Caserta. Em T1 utilizou-se irrigação por gotejamento, com mulching sobre os camalhões e menor aplicação de biocidas que em T2. Em T2 utilizou-se irrigação por aspersores, colocados a 1,8 m de altura, sem mulching sobre os camalhões. Aplicou-se o teste de Bartlett entre as seis variâncias das linhas de cultivo dentro de cada colheita e entre as variâncias médias das seis linhas para verificar a homogeneidade entre colheitas em cada estação de cultivo. Para comparação das médias de produção de cada linha de cultivo, em cada colheita, dentro de cada túnel, e entre linhas de cultivo, com a mesma posição nos túneis, foi aplicado o teste t. Na estação $\mathrm{V} / \mathrm{O}$, as variâncias foram homogêneas em duas das onze colheitas e, na estação I/P, em dez das treze colheitas, sendo que em ambas as estações houve maior homogeneidade das variâncias na primeira metade do ciclo. Na estação V/O, as variâncias em T1 foram, de modo geral, menores em todo o ciclo. Nas duas estações houve heterogeneidade na variância entre colheitas. Na estação $\mathrm{V} / \mathrm{O}$, ocorreram diferenças significativas em seis e três colheitas, respectivamente em T1 e T2, com as médias de produção atingindo 481,0 em T1 e 454,0 $\mathrm{g} \mathrm{planta}^{-1}$ em T2. Na estação I/P ocorreram diferenças de produção na primeira (T1) e quarta e quinta colheitas (T2), com as médias atingindo 598,2 (T1) e 597,4 g planta-1 (T2).
\end{abstract}

Palavras-chave: Curcubita pepo, ambiente protegido, planejamento de experimentos, tamanho de parcelas, homogeneidade de variância.

\section{ABSTRACT \\ Variance and means of zucchini fruit mass in multiple harvests}

The variance associated to the production of plants with multiple harvests is not always homogeneous, what compromises the experimental precision. To address this issue, in this work we identified (1) the behavior of the means and variance for fruit mass in zucchini (Curcubita pepo) along harvests, between planting rows within and betweens harvests, and (2) the interference of different crop managements over these parameters. The experiment was carried out in two seasons, winter-spring (I/P) and summer-autumn (V/O), 2004/ 2005, using cultivar Caserta and two plastic tunnels (T1 and T2), with three 25-plant rows in each tunnel. In T1, drip irrigation was used, combined with mulching over the ridges, and less biocide spraying than in T2. In T2, sprinkler irrigation was used, placed 1,8 m height, without mulching. The Bartlett test was applied to the six planting row variances within each harvest and to the average variance of each row to check the homogeneity between harvests in each crop season. The $t$ test was used to compare the production means in each planting row, in each harvest, in each tunnel, and also between planting rows, for plants with the same position in the tunnel. In the V/O season, variances were homogeneous in two out of the eleven harvests. In season I/P, variances were homogeneous in ten of the thirteen harvests. In both seasons, variance homogeneity was higher in the first half of the cycle. In V/O, variances in T1 were in general smaller than in $\mathrm{T} 2$ in the whole cycle. In both seasons there was variance heterogeneity between harvests. In V/O, significant differences were observed in six and three harvests, respectively in $\mathrm{T} 1$ and $\mathrm{T} 2$, with production means reaching 481.0 and $454.0 \mathrm{~g} \mathrm{plant}^{-1}$ in $\mathrm{T} 1$ and $\mathrm{T} 2$, respectively. In I/P, significant differences were observed in the first (T1) and fourth and fifth harvests (T2), with the means for fruit mass reaching 598.2 (T1) and 597.4 g plant $^{-1}$ (T2).

Keywords: Curcubita pepo, greenhouse, experiment planning, size plot, homogeneity of variances.

\section{(Recebido para publicação em 10 de dezembro de 2006; aceito em 8 de agosto de 2008)}

\begin{abstract}
A abobrinha-italiana (Curcubita pepo), planta da família das cucurbitáceas, situa-se entre as dez hortaliças de maior valor econômico e de maior produção no Brasil. É uma cultura de importância econômica principalmente no centro e sul do País. Tem ciclo de 50 a 80 dias, podendo ser cultivada a campo tanto no verão, quanto na primavera (Camargo, 1981). Em cultivo protegido, além desses períodos,
\end{abstract}

pode ser produzida no outono e inverno, quando o preço por quilo pode triplicar. Com isso, no Rio Grande do Sul, o cultivo da abobrinha-italiana em estufas plásticas é uma alternativa para os produtores aumentarem seus lucros, devido à redução de perdas e ao aumento da produtividade. De acordo com Streck (2002), a produtividade pode alcançar mais que o dobro da relatada por Filgueira (2000), que cita um rendimen- to de cerca de oito toneladas para cultivos a campo, além de apresentar vantagens, principalmente na qualidade superior dos produtos e produção em baixas temperaturas.

A opção de produzir abobrinha-italiana em ambiente protegido acontece em função da grande expansão que esse sistema de cultivo vem experimentando. O uso cada vez mais freqüente de ambientes protegidos deve-se ao melhor 
controle das condições de crescimento e à aceleração de vários aspectos que regulam o crescimento e a maturação das plantas. Paralelamente a essa expansão, aumenta a demanda do setor produtivo por resultados de pesquisa que auxiliem não só na solução dos problemas enfrentados, mas também no incremento da eficiência do sistema. Em conseqüência, surge a necessidade de aumentar a confiabilidade dos resultados de pesquisa obtidos em experimentos conduzidos em ambientes protegidos. Experimentos neste sistema de cultivo, assim como em outras áreas, devem ser bem compreendidos e executados, pois a precisão caracteriza a qualidade das inferências dos resultados.

Os pesquisadores, ao realizarem seus experimentos, esperam que a variabilidade ocorrida entre as parcelas seja atribuída à média e, no caso de parcelas de diferentes tratamentos, ao efeito destes. Porém, por mais cuidado que se tenha, ocorrem sempre variações aleatórias entre as parcelas com mesmo tratamento. Estas variações são denominadas de erro experimental (Steel et al., 1997). Diversas fontes de erro experimental estão presentes em experimentos e aqueles conduzidos em ambiente protegido também são afetados por essas fontes de heterogeneidade, entre elas a heterogeneidade do material experimental utilizado, a aplicação não completamente uniforme de tratos culturais, o ataque de pragas e doenças e as injúrias causadas por sucessivas colheitas na mesma planta (Lorentz et al., 2004, 2005).

As formas de controlar o erro experimental são a utilização adequada do delineamento experimental, a determinação do tamanho e forma das parcelas e o uso de observações auxiliares (Steel et al., 1997). Estas formas de controle experimental vêm sendo estudadas principalmente nas grandes culturas, como milho (Rezende \& Souza Júnior, 1997; Martin et al., 2005b), sorgo (Lopes et al., 2005) e soja (Martin et al., 2005a), enquanto para culturas olerícolas, principalmente aquelas cultivadas em ambientes protegidos, as informações a este respeito são escassas (Marodin et al., 2000; Lúcio et al., 2003; Mello et al., 2004). Atualmente, os trabalhos com olerícolas são realizados, na sua maioria, em parcelas experimentais cujo tamanho foi escolhido empiricamente, determinado pela necessidade ou praticidade ou, ainda, baseado em experiências anteriores, seguindo tendências a partir de trabalhos já realizados. O mesmo acontece com o delineamento, número de repetições e a intensidade de amostragem dentro das parcelas.

Em experimentos conduzidos em cultivo protegido sem aplicação de tratamentos, compostos de linhas de cultivos bem definidas e cujas plantas são tratadas de modo individual e não tendencioso, espera-se que as médias de produção das linhas e suas respectivas variâncias não difiram entre si. No entanto, este último pressuposto pode não estar sendo atendido para os experimentos realizados em estufas plásticas, induzindo a conclusões errôneas a respeito das diferenças entre os tratamentos. Souza et al. (2002) relataram a grande variabilidade existente para crescimento dos frutos de abobrinha-italiana. Da mesma forma, Lúcio et al. (2003) observaram colheitas de pimentão $100 \%$ heterogêneas. Já Lorentz et al. (2004), trabalhando com pepineiro, verificaram que sete em treze das colheitas se mostraram com variâncias homogêneas, observando também grandes oscilações das médias da produção por colheita. Estudando a variabilidade da produção de tomate em função das colheitas, Lopes et al. (1998) verificaram que ocorreram mudanças na concentração da produção no decorrer das colheitas e constataram diferenças de produção entre as linhas de cultivo.

A falta de homogeneidade das variâncias passa a ser problemática quando se utilizam testes de comparação de médias, já que a homogeneidade entre as variâncias é um dos pressupostos para realização da análise da variância e, portanto, necessária para assegurar o nível de significância dos testes $\mathrm{F}$ e de comparação das médias. Em análises de dados experimentais com variâncias heterogêneas, o nível de significância passa a ficar acima do especificado (Conagin et al., 1993). Assim, o estudo do comportamento da média e da variância dentro do ambiente protegido ao longo de diferentes co- lheitas se faz necessário, bem como a interferência direta dos diferentes manejos nessas estatísticas, pois são fatores que podem alterar as conclusões de trabalhos científicos.

Desta forma, os objetivos deste trabalho foram identificar o comportamento da média e da variância da massa dos frutos de abobrinha-italiana com o passar das colheitas, entre as linhas de cultivo dentro de cada colheita e entre colheitas, e verificar a interferência do manejo nesses parâmetros.

\section{MATERIAL E MÉTODOS}

Os experimentos com abobrinha-italiana, cultivar Caserta, foram conduzidos no Departamento de Fitotecnia da Universidade Federal de Santa Maria (2941' $\mathrm{S}, 5^{\circ} 41^{\prime} \mathrm{W}$ e altitude de $95 \mathrm{~m}$ ). O clima é classificado como $\mathrm{Cfa}$, subtropical úmido sem estação seca definida e com verões quentes e, o solo, como Argissolo Vermelho Distrófico Arênico.

Foram utilizados dois túneis com dimensões de $25 \mathrm{~m}$ de comprimento por $4 \mathrm{~m}$ de largura, com 3,5 $\mathrm{m}$ de altura na parte central, coberto com filme de plástico PeBD (Polietileno de baixa densidade) de $200 \mu$ (Figura 1), sendo as plantas em cada túnel submetidas a um manejo cultural específico. No primeiro (T1), foi realizada irrigação por gotejamento e foram utilizados camalhões, com 0,1 m de altura e 0,4 m de largura, com mulching preto de PeDB de $35 \mu$. Neste túnel, as laterais não foram fechadas para irrigação e houve uma menor aplicação de fungicidas e inseticidas em relação ao outro manejo. Foram realizadas desfolhas sempre que necessário para o melhor desenvolvimento da cultura, mas em menor quantidade se comparado ao realizado no outro túnel. No segundo túnel (T2) a irrigação foi realizada utilizando aspersores fixados a 1,8 $\mathrm{m}$ acima do solo e os camalhões permaneceram sem mulching. Para aumentar a uniformidade na distribuição de água, o túnel era totalmente fechado no momento da irrigação. Foram realizadas capinas entre as linhas e dentro das linhas, sendo aplicados fungicidas e inseticidas e efetuadas desfolhas sempre que neces- 
sário. Em ambos os túneis as plantas foram conduzidas em três linhas de cultivo, com 25 plantas por linha e espaçamento entre plantas de $0,8 \mathrm{~m} \mathrm{e}$, entre filas, de $1,2 \mathrm{~m}$.

A produção das mudas foi efetuada via sistema de bandejas de isopor de 144 células, em substrato comercial Plantmax ${ }^{\circledR}$, suspensas sobre armação metálica. A semeadura foi realizada em 28/08/04 e em 25/02/05 no interior de uma estufa-sementeira, com cobertura plástica e irrigação com microaspersores. O transplante foi realizado em 14/09/04 e em 15/03/05 para as estações sazonais inverno-primavera (I/P) e verão-outono (V/O), respectivamente, quando as mudas apresentavam seis folhas definitivas ou $15 \mathrm{~cm}$ de estatura. As plantas que após o transplante apresentavam crescimento inferior às demais foram substituídas por plantas reservas, a fim de assegurar um adequado e homogêneo estande de plantas. Todos os tratos culturais foram realizados com base na recomendação para a cultura (Filgueira, 2000). A unidade básica (UB) foi composta por uma planta e resultou, portanto, em $25 \mathrm{UB}$ linha $^{-1}$. As plantas foram identificadas com o número de ordem da linha e da planta dentro de cada linha. A definição do momento da colheita baseou-se no tamanho dos frutos, onde estes foram colhidos quanto apresentavam comprimento acima de $18 \mathrm{~cm}$, sendo colocados em sacos de papel e identificados, para posterior determinação da massa fresca em balança digital com precisão de um grama.

Em cada colheita realizada, estimouse a média e a variância entre as plantas com produção dentro de cada linha. Aplicou-se o teste de Bartlett (Steel et al., 1997) entre as seis variâncias das linhas de cultivo (três linhas de cultivo em cada túnel) obtidas em cada colheita, para verificar a homogeneidade entre as linhas de cultivo dentro de cada colheita. Posteriormente, aplicou-se o teste de Bartlett entre as variâncias médias das seis linhas em cada colheita, para verificar a homogeneidade entre colheitas em cada estação sazonal de cultivo. Para comparação das médias de produção de cada linha de cultivo, em cada colheita dentro de cada túnel, e

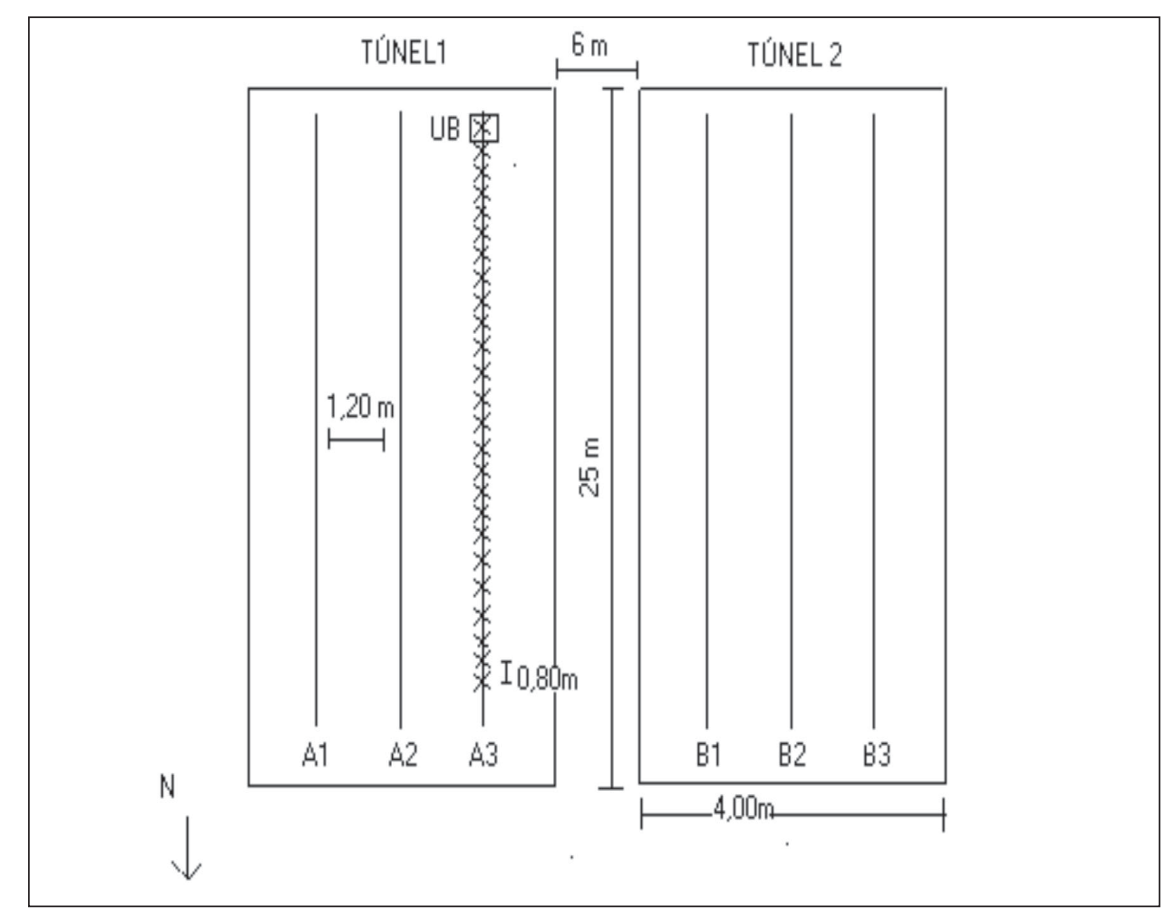

Figura 1. Identificação e localização dos túneis plásticos, linhas de cultivo e unidades básicas (UB) utilizadas no estudo (identification and location of the plastic tunnels, planting rows, and basic units (UB) used in the study). Santa Maria, UFSM, 2005.

entre linhas de cultivo, com a mesma posição nos túneis plásticos, foi aplicado o teste $t$ utilizando as respectivas estimativas de variâncias e graus de liberdade. Em todas as análises estatísticas foi adotado $5 \%$ de probabilidade de erro, utilizando os aplicativos Excel e SAEG, versão 9.1 .

\section{RESULTADOS E DISCUSSÃO}

O teste de Bartlett aplicado nas variâncias das seis linhas (dois túneis), dentro de cada colheita, na estação sazonal verão-outono (V/O), evidenciou que houve homogeneidade em duas (18,2\%) das onze colheitas realizadas. Já na estação sazonal inverno-primavera (I/P), as variâncias foram homogêneas em dez $(76,9 \%)$ das treze colheitas realizadas (Tabela 1). Na estação V/ $\mathrm{O}$, duas das seis primeiras colheitas mostraram-se homogêneas (33\%), enquanto na estação I/P, em três das seis primeiras colheitas, a homogeneidade das variâncias foi detectada (50\%). Isso demonstra que, mesmo mudando a estação de cultivo, houve pouca alteração no comportamento na primeira metade do ciclo, o que pode ter acontecido em função da similaridade entre a tempera- tura média do ar (de 18 a $25^{\circ} \mathrm{C}$ na estação V/O; 15 a $23^{\circ} \mathrm{C}$ na estação I/P) e brilho solar (menos de um a 8,5 horas, na estação V/O; menos um a 11,1 horas, na estação I/P), nas duas estações (Figura 2). Por sua vez, a heterogeneidade observada na fase inicial do experimento pode ser consequiência de variações na semeadura ou na obtenção e transplante das mudas, fato também observado em experimentos com pimentão (Lorentz et al., 2005).

Ao se visualizar a segunda metade do ciclo produtivo, a partir da sétima colheita, houve um comportamento diferenciado entre as variâncias das linhas de cultivo nas diferentes estações sazonais (Tabela 1). Na estação V/O, 100\% das colheitas apresentaram variâncias heterogêneas entre linhas, enquanto na estação I/P 100\% das colheitas apresentaram variâncias homogêneas. As heterogeneidades da estação V/O ocorreram provavelmente em função do decréscimo da temperatura do ar e do brilho solar com o avanço do ciclo de cultivo nesta época. A temperatura média do ar oscilou de 21 para $13^{\circ} \mathrm{C}$ e, o brilho solar, entre menos de 1 e 8,4 horas, entre a sétima e a última colheitas (Figura 2). 
Tabela 1. Número de plantas colhidas e variância da massa dos frutos de abobrinha-italiana dentro da colheita, em cada linha de cultivo, para sistema conduzido em túneis plásticos, em duas estações (number of harvested plants and fruit mass variance within harvests in zucchini, in each planting row, under plastic tunnels, in two stations). Santa Maria, UFSM, 2005.

\begin{tabular}{|c|c|c|c|c|c|c|c|}
\hline \multirow{3}{*}{ Colheitas } & & \multicolumn{6}{|c|}{ Linhas de cultivo } \\
\hline & & \multicolumn{3}{|c|}{ Túnel $1^{1}$} & \multicolumn{3}{|c|}{ Túnel $2^{2}$} \\
\hline & & A1 & A2 & A3 & B1 & B2 & B3 \\
\hline \multicolumn{8}{|c|}{ Verão /Outono } \\
\hline \multirow[t]{2}{*}{1} & $\mathrm{~N}^{*}$ & 5 & 8 & 6 & 4 & 4 & 3 \\
\hline & $\mathrm{S}^{2 * *}$ & $45544,5^{* * *}$ & 2964,0 & 4755,9 & 6492,3 & 1289,7 & 7020,3 \\
\hline \multirow[t]{2}{*}{2} & $\mathrm{~N}$ & 12 & 8 & 5 & 8 & 8 & 3 \\
\hline & $s^{2}$ & $8922,0^{* * *}$ & 10953,4 & 3098,5 & 16699,7 & 42682,2 & 4452,3 \\
\hline \multirow[t]{2}{*}{3} & $\mathrm{~N}$ & 6 & 6 & 4 & 11 & 11 & 4 \\
\hline & $s^{2}$ & $2684,4^{* * *}$ & $47.644,6$ & 3554,7 & 15352,3 & 19961,2 & 17318,0 \\
\hline \multirow[t]{2}{*}{4} & $\mathrm{~N}$ & 7 & 8 & 4 & 14 & 6 & 6 \\
\hline & $s^{2}$ & $9212,1^{* * *}$ & 30937,3 & 2488,7 & 106549,8 & 6226,7 & 2238,8 \\
\hline \multirow[t]{2}{*}{5} & $\mathrm{~N}$ & 6 & 5 & 5 & 3 & 8 & 8 \\
\hline & $\mathrm{s}^{2}$ & $13426,1^{\mathrm{ns}}$ & 6721,3 & 6919,8 & 3728,2 & 13144,3 & 9665,7 \\
\hline \multirow[t]{2}{*}{6} & $\mathrm{~N}$ & 6 & 7 & 4 & 5 & 5 & 5 \\
\hline & $s^{2}$ & $21190,5^{\mathrm{ns}}$ & 6424,3 & 5671,6 & 5188,3 & 7755,7 & 6075,3 \\
\hline \multirow[t]{2}{*}{7} & $\mathrm{~N}$ & 9 & 8 & 5 & 8 & 6 & 6 \\
\hline & $s^{2}$ & $66403,7^{* * *}$ & 10754,0 & 36915,7 & 14558,6 & 4169,1 & 12803,8 \\
\hline \multirow[t]{2}{*}{8} & $\mathrm{~N}$ & 9 & 7 & 6 & 9 & 6 & 5 \\
\hline & $s^{2}$ & $8482,6^{* * *}$ & 25894,9 & 10407,5 & 8695,0 & 11560,6 & 88934,0 \\
\hline \multirow[t]{2}{*}{9} & $\mathrm{~N}$ & 5 & 6 & 8 & 10 & 7 & 7 \\
\hline & $s^{2}$ & $70056,2^{* * *}$ & 2871,6 & 10193,1 & 10001,8 & 4339,5 & 9515,8 \\
\hline \multirow[t]{2}{*}{10} & $\mathrm{~N}$ & 8 & 3 & 5 & 6 & 12 & 7 \\
\hline & $s^{2}$ & $1071,6^{* * *}$ & 1390,3 & 1535,3 & 12025,8 & 5674,5 & 7812,5 \\
\hline \multirow[t]{2}{*}{11} & $\mathrm{~N}$ & 4 & 3 & 10 & 10 & 5 & 10 \\
\hline & $\mathrm{s}^{2}$ & $1172,3^{* * *}$ & 24912,3 & 8039,8 & 24322,0 & 98116,7 & 27687,7 \\
\hline \multicolumn{8}{|c|}{ Inverno/Primavera } \\
\hline \multirow[t]{2}{*}{1} & $\mathrm{~N}$ & 9 & 9 & 6 & 11 & 13 & 2 \\
\hline & $s^{2}$ & $5040,2^{* * *}$ & 41350,2 & 2230,6 & 7278,6 & 4294,6 & 6498,0 \\
\hline \multirow[t]{2}{*}{2} & $\mathrm{~N}$ & 4 & 13 & 5 & 10 & 14 & 8 \\
\hline & $s^{2}$ & $17324,9^{\text {ns }}$ & 7166,7 & 9532,2 & 6227,0 & 7956,2 & 5579,7 \\
\hline 3 & $\mathrm{~N}$ & 3 & 6 & 3 & 6 & 6 & 4 \\
\hline & $s^{2}$ & $3600,3^{\text {ns }}$ & 6328,7 & 8323,0 & 5337,4 & 2434,3 & 3287,0 \\
\hline 4 & $\mathrm{~N}$ & 8 & 8 & 2 & 8 & 7 & 3 \\
\hline & $s^{2}$ & $622,8^{* * *}$ & 4989,1 & 544,5 & 3130,2 & 9038,3 & 3137,3 \\
\hline 5 & $\mathrm{~N}$ & 8 & 10 & 8 & 12 & 14 & 3 \\
\hline & $s^{2}$ & $5607,6^{* * *}$ & 17783,2 & 4809,2 & 1762,1 & 6030,6 & 9312,3 \\
\hline 6 & $\mathrm{~N}$ & 10 & 7 & 2 & 4 & 7 & 2 \\
\hline & $s^{2}$ & $12529,8^{\text {ns }}$ & 6818,0 & 5618,0 & 541,0 & 1994,0 & 3613,0 \\
\hline 7 & $\mathrm{~N}$ & 6 & 7 & 2 & 6 & 5 & 3 \\
\hline & $\mathrm{s}^{2}$ & $15417,7^{\mathrm{ns}}$ & 5302,5 & 7200,0 & 14429,5 & 7157,7 & 6521,3 \\
\hline 8 & $\mathrm{~N}$ & 11 & 8 & 9 & 11 & 9 & 4 \\
\hline & $s^{2}$ & $15923,2^{\mathrm{ns}}$ & 57608,5 & 33316,5 & 34416,3 & 38862,5 & 4576,2 \\
\hline 9 & $\mathrm{~N}$ & 9 & 11 & 7 & 10 & 13 & 4 \\
\hline & $s^{2}$ & $63918,2^{\text {ns }}$ & 64815,0 & 126949,6 & 66794,9 & 39282,2 & 46656,3 \\
\hline 10 & $\mathrm{~N}$ & 7 & 7 & 2 & 8 & 8 & 2 \\
\hline & $s^{2}$ & $4896,1^{\mathrm{ns}}$ & 9202,7 & 5100,5 & 21304,6 & 16508,1 & 6844,5 \\
\hline 11 & $\mathrm{~N}$ & 4 & 2 & 4 & 4 & 7 & 5 \\
\hline & $s^{2}$ & $23337,0^{\text {ns }}$ & 3528,0 & 4010,9 & 13951,5 & 3572,2 & 15323,3 \\
\hline 12 & $\mathrm{~N}$ & 6 & 3 & 5 & 8 & 5 & 2 \\
\hline & $\mathrm{s}^{2}$ & $15522,0^{\text {ns }}$ & 5946,3 & 7943,0 & 10461,1 & 70633,7 & 11400,5 \\
\hline 13 & $\mathrm{~N}$ & 4 & 6 & 4 & 6 & 9 & 2 \\
\hline & $S^{2}$ & $3775,0^{\text {ns }}$ & 2522,0 & 5639,3 & 6416,2 & 6909,2 & 2178,0 \\
\hline
\end{tabular}

* $\mathrm{N}$ = número de plantas colhidas em cada colheita, em cada linha de cultivo (number of plants harvested in each harvest, in each planting row); ${ }^{* *} \mathrm{~s}^{2}$ = variância da massa dos frutos ( $\mathrm{g}$ ) dentro da colheita, em cada linha de cultivo (variance of fruit mass ( $\mathrm{g}$ ) within harvests, in each planting row); ${ }^{* * *}$ Variâncias heterogêneas entre as seis linhas de cultivo, teste de Bartlett, $\mathrm{p}<0.05$ (heterogeneous variances among the six planting rows, Bartlett test, p<0.05); ns: Não significativo (non significant); ${ }^{1}$ Túnel 1: irrigação por gotejamento, camalhões cobertos com mulching preto, laterais sem fechamento para irrigação e menor aplicação de fungicidas e inseticidas (drip irrigation, ridges with black mulching, opened sides during irrigation, and less spraying of biocides); ${ }^{2}$ Túnel 2: irrigação por aspersão, sem mulching e laterais fechadas no momento da irrigação (sprinkler irrigation, no mulching, and opened sides during irrigation). 
Em condições de cultivo menos propícias, como na estação $\mathrm{V} / \mathrm{O}$, mesmo em ambiente protegido as diferenças do manejo podem ser melhor expressas. De um modo geral, as variâncias no túnel 1 foram menores em todo ciclo. Isto se deve a um melhor e mais rápido estabelecimento da cultura em relação ao túnel 2, proporcionando uma produção mais homogênea em condições desfavoráveis de cultivo. Por outro lado, o estabelecimento das plantas no túnel 2 pode ter sido mais lento em decorrência das variações climáticas relatadas, o que reduz a produção inicial, mas resulta em aumento da produção quando todo o ciclo de cultivo é considerado. Já na estação sazonal I/P houve homogeneidade das variâncias dentro de cada colheita. Concomitantemente, observou-se melhoria das condições climáticas no decorrer do experimento, com aumento da temperatura e do brilho solar. Nesta época, a temperatura média do ar oscilou de 20 a $27^{\circ} \mathrm{C}$ e, o brilho solar, de 2 até 12 horas, mas com uma menor variação em relação à outra época, o que torna menos marcantes as diferenças de manejo e melhora conseqüentemente as condições gerais de cultivo (Figura 2).

Observou-se que as duas estações sazonais apresentaram variâncias heterogêneas entre colheitas (Tabela 2), o que se deve principalmente à variabilidade do crescimento dos frutos entre as condições ambientais favoráveis (crescimento rápido) da estação I/P e as condições desfavoráveis (crescimento lento) da estação V/O. As principais condições ambientais que influenciam o crescimento são a temperatura, radiação solar global, nebulosidade e umidade relativa do ar, como também constatado por Souza et al. (2002). A cultura da abobrinha desenvolve-se melhor, de acordo com Filgueira (2000), em climas secos com temperatura entre 18 e $35^{\circ} \mathrm{C}$, com limites mínimo de $10^{\circ} \mathrm{C}$ e máximo de $32^{\circ} \mathrm{C}$ e umidade relativa do ar mediana (40 a 50\%), condições estas observadas na estação $\mathrm{V} / \mathrm{O}$, na segunda metade do ciclo produtivo.

Na comparação das médias entre linhas de cultivo, na estação V/O, dentro de cada túnel (Tabela 3), verificou-se que ocorreu diferença significativa em seis das onze colheitas no túnel 1 e em

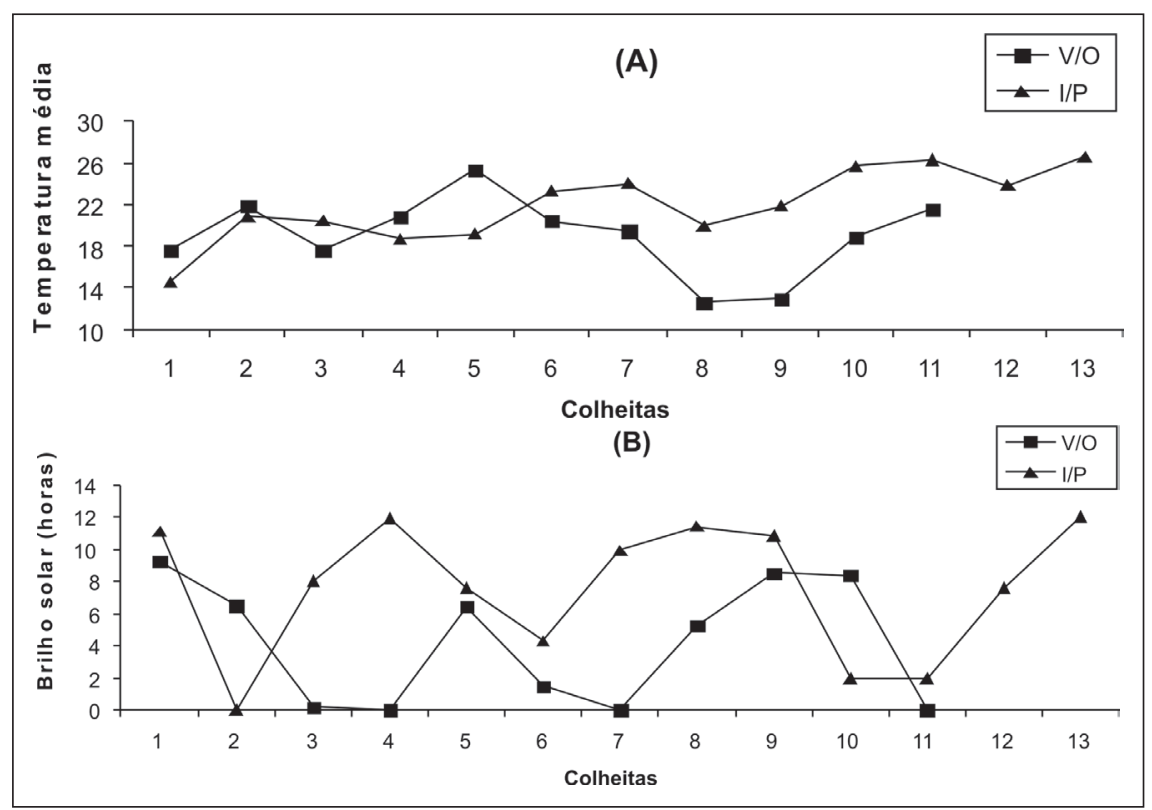

Figura 2. Temperatura média $\left({ }^{\circ} \mathrm{C}\right)(\mathrm{A})$ e brilho solar (B) no decorrer das colheitas de abobrinha-italiana nas estações sazonais verão-outono (V/O) inverno-primavera (I/P) (average temperature $\left({ }^{\circ} \mathrm{C}\right)(\mathrm{A})$ and sunshine $(\mathrm{B})$ along zucchini harvests in summer-autumn $(\mathrm{V} / \mathrm{O})$ and winter-spring (I/P) seasons). Santa Maria, UFSM, 2005.

Tabela 2. Variâncias médias da massa de frutos de abobrinha-italiana entre as plantas das linhas, em cada colheita, para sistema conduzido em túneis plásticos, em duas estações (average variance between plants within the row for zucchini fruit mass, in each harvest, under plastic tunnel, in two seasons). Santa Maria, UFSM, 2005.

\begin{tabular}{|c|c|c|}
\hline Colheitas & $\begin{array}{c}\text { Verão - Outono } \\
\mathrm{s}^{2} \text { massa de frutos }(\mathrm{g})\end{array}$ & $\begin{array}{c}\text { Inverno - Primavera } \\
\mathrm{s}^{2} \text { massa de frutos }(\mathrm{g})\end{array}$ \\
\hline 1 & $11003,8^{*}$ & $11661,3^{*}$ \\
\hline 2 & 16099,7 & 7804,9 \\
\hline 3 & 18538,8 & 6710,5 \\
\hline 4 & 43763,6 & 4074,8 \\
\hline 5 & 9959,4 & 7130,1 \\
\hline 6 & 9138,1 & 6788,2 \\
\hline 7 & 26137,4 & 9996,7 \\
\hline 8 & 21065,8 & 32561,3 \\
\hline 9 & 14569,9 & 65285,5 \\
\hline 10 & 5309,9 & 12901,0 \\
\hline 11 & 27395,9 & 10507,7 \\
\hline 12 & - - - - - & 21236,4 \\
\hline 13 & - & 5215,5 \\
\hline
\end{tabular}

*Variâncias heterogêneas entre colheitas, dentro de cada estação sazonal de cultivo, teste de Bartlett, $\mathrm{p}<0,05$ (heterogeneous variances between harvests within seasons, Bartlett test, $\mathrm{p}<0.05$ ).

três das 11 colheitas no túnel 2, sendo que a linha $\mathrm{A} 2$ apresentou médias superiores em três das seis colheitas e não diferiu da linha A1 em oito das 11 colheitas no túnel 1. Já no túnel 2, a linha B3 foi a que apresentou maior média em três colheitas, diferindo apenas em uma colheita das outras linhas, com diferença significativa das linhas A2 e A1 e ainda B1 e B2 em relação às linhas A3 e
B3. Comparando as linhas com mesma posição dentro de T1 e T2, verificou-se que não houve diferença entre A1 e B1 nas 11 colheitas do V/O. Já para as linhas A2 e B2 a diferença significativa foi verificada apenas na primeira colheita, sendo também observada entre as linhas A3 e B3 na terceira, quarta e sexta colheitas, com um comportamento de maior média de produção na linha A3 
Tabela 3. Produção de frutos de abobrinha-italiana por planta, em cultivo em túneis plásticos com diferentes manejos culturais, em duas estações sazonais de cultivo (production of zucchini fruits per plant, grown under plastic tunnels with different management systems, in two seasons). Santa Maria, UFSM, 2005.

\begin{tabular}{|c|c|c|c|c|c|c|c|c|}
\hline \multirow[b]{2}{*}{ Colheita } & \multicolumn{4}{|c|}{ Túnel $1^{1}\left(\mathrm{~g}_{\text {planta }}{ }^{-1}\right)$} & \multicolumn{4}{|c|}{ Túnel $2^{2}\left(\right.$ g planta $\left.^{-1}\right)$} \\
\hline & A1 & A2 & A3 & Média & B1 & B2 & B3 & Média \\
\hline & \multicolumn{8}{|c|}{ Verão/Outono } \\
\hline 1 & 446,0 a & $308,3 \mathrm{a}$ & $345,6 \mathrm{a}$ & 366,6 & $385,7 \mathrm{a}$ & $403,5 \mathrm{a}$ & 346,6 a & 378,6 \\
\hline 2 & 399,3 a & $390,0 \mathrm{a}$ & $307,0 \mathrm{a}$ & 365,4 & $451,5 \mathrm{a}$ & $511,7 a$ & 352,3 a & 438,5 \\
\hline 3 & $382,0 \mathrm{~b}$ & 616,6 a & $438,0 \mathrm{ab}$ & 382,0 & 400,9 a & $432,2 \mathrm{a}$ & 396,0 a & 409,7 \\
\hline 4 & 459,1 a & $442,8 \mathrm{a}$ & $438,0 \mathrm{a}$ & 446,6 & $531,2 \mathrm{a}$ & $449,3 a b$ & $346,0 \mathrm{~b}$ & 442,1 \\
\hline 5 & $386,8 a b$ & $410,4 \mathrm{a}$ & 277,6 b & 358,3 & $415,6 a b$ & $440,0 \mathrm{a}$ & $286,5 b$ & 380,7 \\
\hline 6 & $419,1 \mathrm{ab}$ & $328,5 \quad b$ & $503,7 \mathrm{a}$ & 417,1 & 514,6 a & $395,0 \mathrm{~b}$ & $295,4 \quad b$ & 401,9 \\
\hline 7 & 527,3 a & $281,2 b$ & $401,2 a b$ & 403,2 & $394,0 \mathrm{a}$ & $388,5 \mathrm{a}$ & 442,3 a & 408,3 \\
\hline 8 & 435,7 a & $413,7 \mathrm{a}$ & 409,5 a & 419,6 & $402,5 \mathrm{a}$ & $371,1 \mathrm{a}$ & $502,0 \mathrm{a}$ & 425,2 \\
\hline 9 & 439,8 a & $333,0 \mathrm{a}$ & 326,7 a & 366,5 & 379,0 b & $406,8 \mathrm{~b}$ & 554,0 a & 397,5 \\
\hline 10 & 279,6 b & $355,6 \mathrm{a}$ & $341,4 \mathrm{a}$ & 325,5 & 403,8 a & 374,7 a & $369,1 \mathrm{a}$ & 382,5 \\
\hline 11 & 605,5 a & $501,6 \mathrm{a}$ & $338,6 \mathrm{~b}$ & 481,9 & $464,3 \mathrm{a}$ & 497,8 a & 399,9 a & 454,0 \\
\hline Média & 434,6 & 398,3 & 375,2 & 393,9 & 431,2 & 424,6 & 390 & 410,8 \\
\hline \multirow{2}{*}{ Colheita } & \multicolumn{8}{|c|}{ Inverno/Primavera } \\
\hline & A1 & A2 & A3 & Média & B1 & B2 & B3 & Média \\
\hline 1 & $433,6 \mathrm{~b}$ & $608,8 \mathrm{a}$ & $440,6 a b$ & 494,3 & $416,5 \mathrm{a}$ & $404,2 \mathrm{a}$ & $389,0 \mathrm{a}$ & 403,2 \\
\hline 2 & 514,7 a & 452,3 a & $449,2 \mathrm{a}$ & 473,1 & 404,9 a & $410,5 a$ & $425,5 \mathrm{a}$ & 413,6 \\
\hline 3 & $442,3 a b$ & $360,5 b$ & 522,3 a & 441,7 & $360,6 a$ & 375,5 a & $321,5 a$ & 352,5 \\
\hline 4 & $323,3 \mathrm{~b}$ & $362,0 a b$ & 445,5 a & 376,9 & 334,6 a & $402,0 \mathrm{a}$ & 303,3 a & 346,6 \\
\hline 5 & 419,3 a & $448,1 \mathrm{a}$ & 436,8 a & 434,7 & $392,0 \mathrm{~b}$ & $446,7 \mathrm{a}$ & $412,6 a b$ & 417,1 \\
\hline 6 & 444,5 a & $478,4 \mathrm{a}$ & $555,0 \mathrm{a}$ & 492,6 & $436,0 \mathrm{a}$ & 417,8 a & 376,5 a & 410,1 \\
\hline 7 & 383,8 a & $411,7 \mathrm{a}$ & $362,0 \mathrm{a}$ & 385,8 & $430,5 \mathrm{a}$ & 410,8 a & 452,6 a & 431,3 \\
\hline 8 & 546,5 a & $638,6 a$ & 508,6 a & 564,6 & 564,8 a & $541,4 \mathrm{a}$ & 490,7 a & 532,3 \\
\hline 9 & 545,3 a & 513,7 a & 735,7 a & 598,2 & 590,9 a & 647,3 a & 554,0 a & 597,4 \\
\hline 10 & 476,8 a & $413,5 a$ & $497,5 \mathrm{a}$ & 462,6 & $487,0 \mathrm{a}$ & $453,8 \mathrm{a}$ & $392,5 \mathrm{a}$ & 444,4 \\
\hline 11 & 526,5 a & $464,0 \mathrm{a}$ & 368,7 a & 453,1 & 485,7 a & $438,4 a$ & 370,4 a & 431,5 \\
\hline 12 & $430,0 \mathrm{a}$ & 489,6 a & $424,0 \mathrm{a}$ & 447,9 & $453,7 \mathrm{a}$ & 554,8 a & 396,5 a & 468,3 \\
\hline 13 & 380,5 a & $404,0 \mathrm{a}$ & $419,0 \mathrm{a}$ & 401,2 & 424,3 a & 459,6 a & $389,0 \mathrm{a}$ & 424,3 \\
\hline Média & 409,4 & 397,8 & 474,2 & 463,6 & 419,8 & 458,7 & 405,7 & 436,4 \\
\hline
\end{tabular}

*Médias seguidas de mesma letra, dentro de cada túnel em cada colheita, não diferem entre si pelo teste t, $\mathrm{p}<0,05$ (means followed by same letter, within each tunnel in each crop, did not differ from each other, $t$ test, $p<0.05$ ); ${ }^{1 / T u ́ n e l ~ 1: ~ i r r i g a c ̧ a ̃ o ~ p o r ~ g o t e j a m e n t o, ~ c a m a l h o ̃ e s ~}$ cobertos com mulching preto, laterais sem fechamento para irrigação e menor aplicação de fungicidas e inseticidas (drip irrigation, ridges with black mulching, opened sides during irrigation, and less spraying of biocides); ${ }^{2} /$ Túnel 2: irrigação por aspersão, sem mulching e laterais fechadas no momento da irrigação (sprinkler irrigation, no mulching, and opened sides during irrigation).

do que a linha B3. Na segunda metade do ciclo produtivo no túnel 2 , as médias das colheitas foram maiores pelo fato das plantas do túnel 2 terem sofrido mais com as variações de temperatura no início do ciclo, que vêm ao encontro do comportamento das variâncias.

Já na comparação entre as médias nas linhas de cultivo nas treze colheitas na estação sazonal I/P dentro de cada túnel (Tabela 3), ocorreu diferença entre as médias na primeira colheita do túnel 1 e na quarta e quinta colheitas do túnel 2, mas sempre os menores valores de médias estavam na primeira ou terceira linhas dos túneis. Isso evidencia melhores condições na linha do meio em relação à das extremidades. Já no túnel 2 , na segunda metade do ciclo produtivo, as médias da linha B3 tiveram, na sua maioria, valores menores em relação às outras duas linhas. Na comparação entre as linhas A1 e B1, ocorreu diferença significativa na terceira e na décima colheitas; entre as linhas A2 e $\mathrm{B} 2$, na primeira, terceira, sétima e nona colheitas e; entre A3 e B3, na quarta, sexta e nona colheitas, sendo que a linha A3 do túnel 1 atingiu a maior média nas quarta e sexta colheitas. Estas diferenças ocorreram pela localização das linhas, tanto no túnel 1 , quanto no túnel 2, o que foi agravado pela diferença de manejo, demonstrando que as linhas na posição A3 e B3 foram as mais prejudicadas. Esse resultado são concordantes com Feijó et al. (2005) que observou um menor desenvolvimento das plantas localizadas na fila oeste, pois, por haver grande intensidade de ventos no lado leste, este permaneceu com as laterais fechadas, enquanto o lado oeste permaneceu com as laterais abertas, o que contribuiu para o não tombamento das plantas de abobrinha-italiana.

As diferenças apresentadas entre as linhas de cultivo, dentro de cada túnel e entre linhas com a mesma posição em 
túneis diferentes, mostram que com o passar do ciclo e as múltiplas colheitas realizadas, as plantas vão expressandose de forma diferenciada, sendo influenciadas diretamente pelas condições climáticas, pelo tipo de manejo, bem como pela proximidade da linha de cultivo com as laterais do túnel plástico e a posição daquela na área cultivada. Em condições de limitações de temperatura do ar e brilho solar, as plantas nas linhas com camalhões sem mulching e com recebimento de água na irrigação por aspersão, tendem a apresentar variâncias heterogêneas e diferenças significativas entre médias, comportamento este não evidenciado em condições favoráveis ao crescimento e desenvolvimento das plantas.

Tanto as médias quanto às variâncias do peso da massa de frutos de abobrinha italiana oscilaram de forma significativa entre as linhas de cultivo com o passar do ciclo produtivo e das múltiplas colheitas realizadas, independente da estação sazonal de cultivo. Plantas de abobrinha-italiana, cultivadas em sistema de irrigação por aspersão e sem cobertura do camalhão por mulching, tendem a apresentar médias e variâncias significativamente diferentes entre as linhas de cultivo, quando em condições de limitação de fatores climáticos como temperatura do ar e horas de brilho solar.

\section{AGRADECIMENTOS}

Ao CNPq e FAPERGS/RS, pelos recursos financeiros para a realização do trabalho e bolsas de produtividade em pesquisa e de iniciação científica.

\section{REFERÊNCIAS}

CAMARGO LS. 1981. As hortaliças e seu cultivo. Campinas: Fundação Cargill. 321p.

CONAGIN A; NAGAI V; IGUE T. 1993. Efeito da falta de normalidade em testes de homogeneidade das variâncias. Bragantia 57: 203-214.

FEIJÓ S; OLIVEIRA SJR; STORCK L; LÚCIO AD; DAMO HP; MARTINI LFD. 2005. Repetibilidade da produção de frutos de abobrinha italiana. Revista Brasileira de Agrociência 11: 39-43.

FILGUEIRA FAR. 2000. Novo manual de Olericultura: tecnologia moderna na produção e comercialização de hortaliças. Viçosa: UFV. 402p.

LOPES SJ; STORCK L; HELDWEIN AB; FEIJÓ S; ROS CA. 1998. Técnicas experimentais para tomateiro tipo salada sob estufas plásticas. Ciência Rural 28: 193-197.

LOPES SJ; STORCK L; LÚCIO AD; LORENTZ LH; LOVATO C; DIAS VO. 2005. Tamanho de parcela para produtividade de grãos de sorgo granífero em diferentes densidades de plantas. Pesquisa Agropecuária Brasileira 40: 525530.

LORENTZ LH; LÚCIO AD; STORCK L; LOPES SJ; BOLIGON AA; CARPES RH. 2004. Variação temporal do tamanho de amostra para experimentos em estufa plástica. Ciência Rural 34: 1043-1049.

LORENTZ LH; LÚCIO AD; BOLIGON AA; LOPES SJ; STORCK L. 2005. Variabilidade da produção de frutos de pimentão em estufa plástica. Ciência Rural 35: 316-323.

LÚCIO AD; SOUZA MF; HELDWEIN AB; LIEBERKNECHT D; CARPES RH; CARVALHO MP. 2003. Tamanho da amostra e método de amostragem para avaliação de características do pimentão em estufa plástica. Horticultura Brasileira 21: 181-185.
MARTIN TN; DUTRA LMC; JAUER A; STORCK L. 2005a. Tamanho ótimo de parcela e número de repetições em soja (Glycine max). Ciência Rural 35: 271-276.

MARTIN TN; STORCK L; LÚCIO AD; CARVALHO MP; SANTOS PM. 2005b. Bases genéticas de milho e alterações no plano experimental. Pesquisa Agropecuária Brasileira 40: 35-40.

MELLO RM; LÚCIO AD; STORCK L; LORENTZ LH; CARPES RH; BOLIGON AA. 2004. Size and form of plots for the culture of the Italian Pumpkin in plastic greenhouse. Scientia Agricola 61: 457-461.

MARODIM VS; STORCK L; LOPES SJ. 2000. Delineamento experimental e tamanho de amostra para alface cultivada em hidroponia. Ciência Rural 30: 779-781.

REZENDE MDV; SOUZA JÚNIOR CL. 1997. Número de repetições e tamanho de parcela para seleção de progênies de milho em solos sob cerrado e fértil. Pesquisa Agropecuária Brasileira 32: 781-788.

SOUZA MF; LÚCIO AD; STORCK L; CARPES RH; SANTOS PM; SIQUEIRA LF. 2002. Tamanho da amostra para peso da massa de frutos, na cultura da abóbora italiana em estufa plástica. Revista Brasileira de Agrociência 8: 123-128.

STEEL RGD; TORRIE JH; DICKEY DA. 1997. Principles and procedures of statistics: a biometrical approach. New York: McGrawHill. 666p.

STRECK L. 2002. Determinação e modelização da evapotranspiração máxima e do coeficiente de cultura da abóbora italiana em estufa plástica. Santa Maria: Universidade Federal de Santa Maria. 92p. (Dissertação de Mestrado) 